\title{
Maximal regularity for the Lamé system in certain classes of non-smooth domains
}

\author{
Marius Mitrea* and Sylvie Monniaux
}

November 14, 2010

\begin{abstract}
The aim of this article is twofold. On the one hand, we study the analyticity of the semigroup generated by the Lamé system $-\mu \Delta-\mu^{\prime} \nabla$ div in $L^{q}(\Omega)$, where $\Omega$ is an open subset of $\mathbb{R}^{n}$ satisfying mild regularity assumptions and the Lamé moduli $\mu, \mu^{\prime}$ are such that $\mu>0$ and $\mu+\mu^{\prime}>0$. On the other hand, we use the aforementioned result to prove a maximal regularity property for the time-dependent Lamé system equipped with a homogeneous Dirichlet boundary condition.
\end{abstract}

\section{Introduction}

Let $\Omega \subset \mathbb{R}^{n}$ be a bounded open set and fix $\mu, \mu^{\prime} \in \mathbb{R}$. Under mild regularity conditions on $\Omega$ (of geometric measure theoretic type, to be imposed later) and certain algebraic conditions on the Lame moduli $\mu, \mu^{\prime}$, here we propose to prove a maximal regularity result for the Lamé system equipped with Dirichlet boundary conditions

$$
\begin{aligned}
\partial_{t} u-\mu \Delta u-\mu^{\prime} \nabla \operatorname{div} u & =f, & & (t, x) \in(0,+\infty) \times \Omega, \\
u(0) & =0, & & x \in \Omega, \\
u(t, x) & =0, & & (t, x) \in[0,+\infty) \times \partial \Omega .
\end{aligned}
$$

More specifically, the goal is to show that whenever $f \in L^{p}\left(0, \infty ; L^{q}\left(\Omega ; \mathbb{R}^{n}\right)\right)$, the solution $u$ of the system (1.1) belongs to $W^{1, p}\left(0, \infty ; L^{q}\left(\Omega ; \mathbb{R}^{n}\right)\right)$, for certain values of $q$ depending of the nature of the domain $\Omega$, and for all $p>1$. This question was suggested by Raphaël Danchin in connection with the study of the compressible Navier-Stokes system, see [6].

\footnotetext{
* Supported in part by the US NSF grants DMS - 0400639 and DMS-FRG - 0456306 This work has been carried out at Université Aix-Marseille 3, France, and the first-named author gratefully acknowledges the hospitality and support offered by this institution.

2000 Mathematics Subject Classification. Primary: 47D06, 35J50; Secondary: 47D03, 35J55, 49Q15

Key words and phrases. Lamé system, non-smooth domain, maximal regularity
} 


\subsection{The $L^{2}$ setup}

Explaining the initial setup, which is based on classical variational methods, requires that we first define an appropriate version of the three-dimensional curl operator in $\mathbb{R}^{n}$.

Definition 1.1. Denote by $\mathscr{D}^{\prime}(\Omega)$ the space of distributions in an open set $\Omega \subseteq \mathbb{R}^{n}$, and let $\mathscr{D}^{\prime}\left(\Omega ; \mathbb{R}^{m}\right):=\mathscr{D}^{\prime}(\Omega) \otimes \mathbb{R}^{m}$ stand for the collection of $\mathbb{R}^{m}$ valued distributions in $\Omega$. For a distribution $u \in \mathscr{D}^{\prime}\left(\Omega ; \mathbb{R}^{n}\right)$, then define

$$
\operatorname{curl} u:=\left(\frac{1}{\sqrt{2}}\left(\partial_{\ell} u_{\alpha}-\partial_{\alpha} u_{\ell}\right)\right)_{1 \leq \ell, \alpha \leq n}=\frac{1}{\sqrt{2}}\left(\nabla u-(\nabla u)^{\top}\right)
$$

where $\nabla u$ is the Jacobian matrix of first-order partial derivatives (considered in the sense of distributions) of the components of $u$, and the superscript $\top$ denotes (here) the transposition of matrices.

In the context of the above definition, it is then straightforward to check that $\operatorname{curl}^{\top}$, the formal transpose of the curl operator (1.2), acts on a matrix-valued distribution $w=\left(w_{\ell, \alpha}\right)_{1 \leq \ell, \alpha \leq n} \in \mathscr{D}^{\prime}\left(\Omega ; \mathbb{R}^{n \times n}\right)$ according to

$$
\left(\operatorname{curl}^{\top} w\right)_{1 \leq \ell \leq n}=\frac{1}{\sqrt{2}} \partial_{\alpha}\left(w_{\ell \alpha}-w_{\alpha \ell}\right) \in \mathscr{D}^{\prime}\left(\Omega ; \mathbb{R}^{n}\right)
$$

where the repeated index summation convention is used (here, it is understood that the right-hand side is summed over $\alpha$ ). For any vector-valued distribution $u \in \mathscr{D}^{\prime}\left(\Omega ; \mathbb{R}^{n}\right)$ we then have

$$
\begin{aligned}
\left(\operatorname{curl}^{\top}(\operatorname{curl} u)\right)_{\ell} & =\frac{1}{\sqrt{2}} \partial_{\alpha}\left((\operatorname{curl} u)_{\ell \alpha}-(\operatorname{curl} u)_{\alpha \ell}\right)=\sqrt{2} \partial_{\alpha}(\operatorname{curl} u)_{\ell \alpha} \\
& =\partial_{\ell} \partial_{\alpha} u_{\alpha}-\partial_{\alpha} \partial_{\alpha} u_{\ell}=(\nabla \operatorname{div} u-\Delta u)_{\ell}
\end{aligned}
$$

for every $\ell \in\{1, \ldots, n\}$. Therefore, we obtain the higher-dimensional version of a well-known formula in $\mathbb{R}^{3}$, to the effect that

$$
\operatorname{curl}^{\top} \operatorname{curl} u=\nabla \operatorname{div} u-\Delta u \quad \forall u \in \mathscr{D}^{\prime}\left(\Omega ; \mathbb{R}^{n}\right) .
$$

To proceed, consider next the following sesqui-linear form

$$
a_{D}(u, v):=\mu \int_{\Omega} \operatorname{curl} u \cdot \overline{\operatorname{curl} v} d x+\left(\mu+\mu^{\prime}\right) \int_{\Omega} \operatorname{div} u \overline{\operatorname{div} v} d x,
$$

for $u, v \in H_{0}^{1}\left(\Omega ; \mathbb{R}^{n}\right)$ (defined as the closure of $\mathscr{C}_{c}^{\infty}\left(\Omega ; \mathbb{R}^{n}\right)$ in the vector-valued $L^{2}$-based Sobolev space of order one $\left.H^{1}\left(\Omega ; \mathbb{R}^{n}\right)\right)$. This sesqui-linear form is closed, continuous, as well as coercive if

$$
\mu>0 \text { and } \mu+\mu^{\prime}>0 \text {. }
$$

Indeed, based on the identity (1.5) and successive integrations by parts one readily obtains

$$
\begin{aligned}
a_{D}(u, u) & \geq \min \left\{\mu, \mu+\mu^{\prime}\right\} \int_{\Omega}\left(|\operatorname{curl} u|^{2}+|\operatorname{div} u|^{2}\right) d x \\
& =\min \left\{\mu, \mu+\mu^{\prime}\right\} \int_{\Omega}|\nabla u|^{2} d x,
\end{aligned}
$$


for all $u \in H_{0}^{1}\left(\Omega ; \mathbb{R}^{n}\right)$. Therefore, if $H^{-1}\left(\Omega ; \mathbb{R}^{n}\right):=\left(H_{0}^{1}\left(\Omega ; \mathbb{R}^{n}\right)\right)^{*}$, then the form $a_{D}$ generates a self-adjoint bounded invertible operator

$$
L_{D, 0}: H_{0}^{1}\left(\Omega ; \mathbb{R}^{n}\right) \longrightarrow H^{-1}\left(\Omega ; \mathbb{R}^{n}\right)
$$

It is not difficult to see that $L_{D, 0}$ is given by

$$
L_{D, 0} u=-\mu \Delta u-\mu^{\prime} \nabla \operatorname{div} u, \quad \forall u \in H_{0}^{1}\left(\Omega ; \mathbb{R}^{n}\right) .
$$

Indeed, with $X^{*}\langle\cdot, \cdot\rangle_{X}$ denoting the duality pairing between functionals in the dual $X^{*}$ (of a Banach space $X$ ) and vectors in $X$, for every $u, v \in H_{0}^{1}\left(\Omega ; \mathbb{R}^{n}\right)$ we may compute

$$
\begin{aligned}
H_{H^{-1}}\left\langle L_{D, 0} u, v\right\rangle_{H_{0}^{1}} & =a_{D}(u, v) \\
& ={ }^{2}\langle\mu \nabla u, \nabla v\rangle_{L^{2}}+{ }_{L^{2}}\left\langle\mu^{\prime} \operatorname{div} u, \operatorname{div} v\right\rangle_{L^{2}} \\
& =H^{-1}\langle-\mu \Delta u, v\rangle_{H_{0}^{1}}+H_{H^{-1}}\left\langle-\mu^{\prime} \nabla \operatorname{div} u, v\right\rangle_{H_{0}^{1}} \\
& =H^{-1}\left\langle\left(-\mu \Delta u-\mu^{\prime} \nabla \operatorname{div} u\right), v\right\rangle_{H_{0}^{1}} .
\end{aligned}
$$

We denote by $L_{D}$ the part of $L_{D, 0}$ in $L^{2}\left(\Omega ; \mathbb{R}^{n}\right)$. Concretely, $L_{D}$ is the unbounded linear operator on $L^{2}\left(\Omega ; \mathbb{R}^{n}\right)$ defined by

$$
\begin{gathered}
D\left(L_{D}\right):=\left\{u \in H_{0}^{1}\left(\Omega ; \mathbb{R}^{n}\right): \mu \Delta u+\mu^{\prime} \nabla \operatorname{div} u \in L^{2}\left(\Omega ; \mathbb{R}^{n}\right)\right\} \\
L_{D} u:=-\mu \Delta u-\mu^{\prime} \nabla \operatorname{div} u, \quad \forall u \in D\left(L_{D}\right) .
\end{gathered}
$$

By standard arguments, $-L_{D}$ generates an analytic semigroup in $L^{2}\left(\Omega ; \mathbb{R}^{n}\right)$.

\subsection{Statements of main results}

We begin by specifying one of the basic geometric measure theoretic assumptions on the domain $\Omega \subset \mathbb{R}^{n}$.

Definition 1.2. An open set $\Omega \subset \mathbb{R}^{n}$ is said to satisfy an interior ball condition (henceforth abbreviated I.B.C.) if there exists a positive constant $c$ such that for all $x \in \Omega$ and all $0<r<\frac{1}{2} \operatorname{diam} \Omega$,

$$
\left|B_{\Omega}(x, r)\right| \geq c r^{n}, \quad \text { where } \quad B_{\Omega}(x, r):=\{y \in \Omega:|x-y|<r\} .
$$

The notation $|E|$ for a Lebesgue measurable subset $E$ of $\mathbb{R}^{n}$ stands for the $n$-dimensional Lebesgue measure of the set $E$.

The I.B.C. is closely related to the concept of $d$-sets with $d=n$ (in the terminology of [11], p. 28). Also, the I.B.C. holds in the class of Lipschitz domains or, more generally, for domains satisfying an interior corkscrew condition (cf. [9], Section 3, p. 93). The role of I.B.C. is to ensure that $\Omega$, when equipped with the $n$-dimensional Lebesgue measure $\lambda_{n}$ (induced by $\mathbb{R}^{n}$ ) and the standard Euclidean distance, becomes a space of homogeneous type (in the sense of 《⿶). Indeed, I.B.C. implies that the measure $\lambda_{n}$ is doubling, i.e. there exists a positive constant $c$ such that $\lambda_{n}\left(B_{\Omega}(x, 2 r)\right) \leq c \lambda_{n}\left(B_{\Omega}(x, r)\right)$ whenever $x \in \Omega$ and $0<r<\frac{1}{2} \operatorname{diam} \Omega$. In particular, the version of the Hardy-Littlewood maximal operator adapted to $\Omega$, acting on $f \in L_{l o c}^{1}(\Omega)$ according to

$$
M_{\Omega}(f)(x):=\sup _{0<r<\operatorname{diam} \Omega} \frac{1}{\left|B_{\Omega}(x, r)\right|} \int_{B_{\Omega}(x, r)}|f(y)| d y, \quad \forall x \in \Omega,
$$


is bounded in $L^{p}(\Omega)$ for all $p \in(1, \infty)$ (cf. [1], p. 637).

In preparation for stating the main results of this paper, we review several other definitions and notational conventions. First, given $p \in(1, \infty)$, we let $W_{0}^{1, p}\left(\Omega ; \mathbb{R}^{n}\right)$ be the closure of $\mathscr{C}_{c}^{\infty}\left(\Omega ; \mathbb{R}^{n}\right)$ in $W^{1, p}\left(\Omega ; \mathbb{R}^{n}\right)$, the vector-valued version of the standard $L^{p}$-based Sobolev space of order one, and $W^{-1, p}\left(\Omega ; \mathbb{R}^{n}\right):=$ $\left\{f=f_{0}+\partial_{j} f_{j}: f_{0}, f_{j} \in L^{p}\left(\Omega ; \mathbb{R}^{n}\right)\right\}$, equipped with the usual infimum-type norm (over all representations $f=f_{0}+\partial_{j} f_{j}$ ). Also, for $q \in(1, \infty)$, we consider the following Poisson problem for the Lamé system

$$
\left\{\begin{array}{l}
\mu \Delta u+\mu^{\prime} \nabla \operatorname{div} u=f \in W^{-1, q}\left(\Omega ; \mathbb{R}^{n}\right) \text { in } \Omega, \\
u \in W_{0}^{1, q}\left(\Omega ; \mathbb{R}^{n}\right) .
\end{array}\right.
$$

Next, if $p \in(1, \infty)$, then $p^{\prime}$ denotes the Hölder conjugate exponent of $p$, i.e., $p^{\prime} \in(1, \infty)$ satisfies $\frac{1}{p}+\frac{1}{p^{\prime}}=1$. We will often abbreviate $\|\cdot\|_{L^{p}\left(\Omega ; \mathbb{R}^{n}\right)}$ and $\|\cdot\|_{L^{p}(\Omega)}$ simply by $\|\cdot\|_{p}$. Going further, given $p \in(1, n)$ we set $p^{*}:=\frac{n p}{n-p}$ so that $W^{1, p}(\Omega) \hookrightarrow L^{p^{*}}(\Omega)$ and $L^{p}(\Omega) \hookrightarrow W^{-1, p^{*}}(\Omega)$ whenever $\Omega \subset \mathbb{R}^{n}$ is a bounded open set satisfying an I.B.C. (see [7]). If $p \geq n$, we make the convention that $p^{*}:=+\infty$. Finally, for $p>\frac{n}{n-1}$, we denote by $p_{*}$ the exponent $p_{*}:=\frac{n p}{n+p}$, so that $L^{p_{*}}(\Omega) \hookrightarrow W^{-1, p}(\Omega)$ if $\Omega \subset \mathbb{R}^{n}$ bounded open set satisfying an I.B.C.

We are now in a position to state the following results dealing, respectively, with the analyticity of the semigroup generated by the Lamé operator and the maximal regularity property for the Lamé system equipped with (homogeneous) Dirichlet boundary condition.

Theorem 1.3 (Analytic semigroup). Let $\Omega$ be a bounded open subset of $\mathbb{R}^{n}$ satisfying the interior ball condition (1.13). Suppose that (1.7) holds and assume that $q_{0} \geq 2$ is such that the Poisson problem for the Lamé system (1.15) is wellposed for $q=q_{0}$ (for more on this see Theorem 4.1 below).

Then the unbounded operator $L_{D}$ in $L^{2}\left(\Omega ; \mathbb{R}^{n}\right)$ given in $(1.12)$ extends to an unbounded operator $L_{D}^{q}$ in $L^{q}\left(\Omega ; \mathbb{R}^{n}\right)$ for all $q \in\left[\left(q_{0}^{*}\right)^{\prime}, q_{0}^{*}\right]$, and $-L_{D}^{q}$ generates an analytic semigroup in $L^{q}\left(\Omega ; \mathbb{R}^{n}\right)$.

Theorem 1.4 (Maximal regularity). Let $\Omega$ be a bounded open subset of $\mathbb{R}^{n}$ satisfying the interior ball condition (1.13). Suppose that (1.7) holds and assume that $q_{0} \geq 2$ is such that the Poisson problem for the Lamé system (1.15) is wellposed for $q=q_{0}$ (for more on this see Theorem 4.1 below).

Then the Lamé system $(1.1)$ has the maximal $L^{p}$-regularity property in $L^{q}\left(\Omega ; \mathbb{R}^{n}\right)$ for all $p \in(1, \infty)$ and all $q \in\left(\left(q_{0}^{*}\right)^{\prime}, q_{0}^{*}\right)=: I_{0}$. In other words, for all $p \in(1, \infty)$ and all $q \in I_{0}$, the following holds: for every function $f \in L^{p}\left(0, \infty ; L^{q}\left(\Omega ; \mathbb{R}^{n}\right)\right)$, the system (1.1) has a unique solution u satisfying

$$
\begin{gathered}
\left\|\frac{\partial u}{\partial t}\right\|_{L^{p}\left(0, \infty ; L^{q}\left(\Omega ; \mathbb{R}^{n}\right)\right)}+\left\|\mu \Delta u+\mu^{\prime} \nabla \operatorname{div} u\right\|_{L^{p}\left(0, \infty ; L^{q}\left(\Omega ; \mathbb{R}^{n}\right)\right)} \\
\leq C_{p, q}\|f\|_{L^{p}\left(0, \infty ; L^{q}\left(\Omega ; \mathbb{R}^{n}\right)\right) .}
\end{gathered}
$$

Remark 1.5. For any open set $\Omega$ in $\mathbb{R}^{n}$, the problem (1.15) is always well-posed for $q=2$. As a result, the interval $I_{0}$ in Proposition 1.3 and Theorem 1.4 always contains $\left(\frac{2 n}{n+2}, \frac{2 n}{n-2}\right)$, which gives the maximal interval $(1, \infty)$ in dimension 2 .

Remark 1.6. We wish to single out two particular cases, of independent interest, covered by Theorem 4.1 . 
(i) If $n=3$ and $\Omega$ is a bounded Lipschitz domain, then the interval $I_{0}$ can be taken to be $(1, \infty)$.

(ii) If $n \geq 4$ and $\Omega$ is a bounded $\mathscr{C}^{1}$ domain, then the interval $I_{0}$ can be taken to be $(1, \infty)$.

There is a large volume of work devoted to the study of the two main themes of the current paper, i.e., analytic semigroup and maximal regularity properties for a variety of partial differential equations. Here we only wish to mention the excellent surveys [1], 14], where the interested reader can also find a pedantic exposition of recent work related to maximal regularity as well as a wealth of pertinent references to this subject. In this vein, see also the very recent expository article [22]. Our approach here, which is based on off-diagonal estimates and elliptic regularity results, is an adaptation of our work in 19 .

The plan of the remainder of the paper is as follows. In Section 2 we carry out the proofs of Theorem 1.3 and Theorem 1.4. Section 3 contains a discussion of the methods employed in $\S 2$ from the perspective of general second-order elliptic systems. Finally, Section 1 is devoted to presenting a collection of regularity results for the Poisson problem for the Lamé system in the context of Sobolev spaces in non-smooth domains.

\section{Proofs of main results}

This section is devoted to presenting the proofs of Proposition 1.3 and Theorem 1.4. For an arbitrary, fixed angle $\theta \in(0, \pi)$, consider the sector

$$
\Sigma_{\theta}:=\{z \in \mathbb{C} \backslash\{0\}:|\arg z|<\pi-\theta\} \subset \mathbb{C},
$$

and note that, generally speaking, $|z a+b| \approx|z| a+b$, uniformly for $z \in \Sigma_{\theta}$ and $a, b \geq 0$. We will work under the following hypotheses.

Hypotheses. Let $\Omega \subset \mathbb{R}^{n}$ be a bounded open set with I.B.C. Consider an arbitrary $z \in \Sigma_{\theta}$ and set

$$
t:=\frac{1}{\sqrt{|z|}}=|z|^{-\frac{1}{2}} .
$$

We begin in earnest with the proof of Theorem 1.3. Consider an arbitrary, fixed function $f \in \mathscr{C}_{c}^{\infty}\left(\Omega ; \mathbb{R}^{n}\right) \hookrightarrow L^{2}\left(\Omega ; \mathbb{R}^{n}\right)$ and, with $I$ denoting the identity operator, define

$$
u:=\left(z I+L_{D}\right)^{-1} f \in D\left(L_{D}\right) \hookrightarrow L^{2}\left(\Omega ; \mathbb{R}^{n}\right) .
$$

Next, fix an arbitrary point $x \in \Omega$ along with a partition of unity $\left\{\eta_{j}\right\}_{j \in \mathbb{N}}$ of $\mathbb{R}^{n}$ such that

$$
\begin{gathered}
\eta_{0} \in \mathscr{C}_{c}^{\infty}(B(x, 2 t) ; \mathbb{R}), \quad \eta_{j} \in \mathscr{C}_{c}^{\infty}\left(B\left(x, 2^{j+1} t\right) \backslash B\left(x, 2^{j-1} t\right) ; \mathbb{R}\right), \\
0 \leq \eta_{j} \leq 1, \quad\left|\nabla \eta_{j}\right| \leq \frac{1}{2^{j-1} t}, \quad \sum_{j=0}^{\infty} \eta_{j}=1 \text { in } \mathbb{R}^{n},
\end{gathered}
$$


where $B(x, r)$ is the ball in $\mathbb{R}^{n}$ with center at $x \in \mathbb{R}^{n}$ and radius $r>0$. To proceed, decompose

$$
\begin{aligned}
& f=\sum_{j=0}^{\infty} f_{j}, \quad f_{j}:=\eta_{j} f, \quad u=\sum_{j=0}^{\infty} u_{j}, \\
& \text { where } u_{j}:=\left(z I+L_{D}\right)^{-1} f_{j} \in D\left(L_{D}\right) \text { for } j \in \mathbb{N} .
\end{aligned}
$$

The off-diagonal estimates contained in Proposition 2.1 below constitute the key technical ingredient in the proof of Theorem 1.3.

Proposition 2.1. Granted the above conventions and assumptions, for all $q \in$ $\left[2, q_{0}\right]$, where $q_{0}$ is such that the elliptic Lamé system (1.15) is well-posed for $q=q_{0}$, there exist two constants $C, c>0$ with the property that

$$
|z|\left[\int_{B_{\Omega}(x, t)}\left|u_{j}\right|^{q} d y\right]^{\frac{1}{q}} \leq C e^{-c 2^{j}} t^{n\left(\frac{1}{q}-\frac{1}{2}\right)}\left[\int_{\Omega}\left|f_{j}\right|^{2} d y\right]^{\frac{1}{2}} \quad \forall j \in \mathbb{N},
$$

and

$$
\sqrt{|z|}\left(\int_{B_{\Omega}(x, t)}\left|\nabla u_{j}\right|^{q} d y\right)^{\frac{1}{q}} \leq C e^{-c 2^{j}} t^{n\left(\frac{1}{q}-\frac{1}{2}\right)}\left(\int_{\Omega}\left|f_{j}\right|^{2} d y\right)^{\frac{1}{2}}, \quad \forall j \in \mathbb{N} .
$$

In order to facilitate the proof of this proposition, we will first deal with the following iteration lemma.

Lemma 2.2. Retain the same hypotheses and conventions as before, and let $q \in\left[2, q_{0}\right]$ be such that (2.8) and (2.9) hold. Then also (2.8) holds with $q^{*}=\frac{n q}{n-q}$ in place of $q$. Furthermore, if $q^{*} \leq q_{0}$ then (2.9) also holds with $q^{*}$ in lieu of $q$.

Proof. We first prove that (2.8) and (2.9) for $q$ imply (2.8) for $q^{*}$. To this end, recall that Sobolev's embedding in a reasonable domain $D \subset \mathbb{R}^{n}$ of diameter $R>0$, for a function $\varphi \in W^{1, q}\left(\mathbb{R}^{n}\right)$, after rescaling, reads as follows

$$
R^{n\left(\frac{1}{q}-\frac{1}{q^{*}}\right)}\left(\int_{D}|\varphi|^{q^{*}} d y\right)^{\frac{1}{q^{*}}} \leq C\left[\left(\int_{D}|\varphi|^{q} d y\right)^{\frac{1}{q}}+R\left(\int_{D}|\nabla \varphi|^{q} d y\right)^{\frac{1}{q}}\right] .
$$

By Theorem 4.1 and assumptions, we have $u_{j} \in W_{0}^{1, q}\left(\Omega ; \mathbb{R}^{n}\right)$. Let $\tilde{u}_{j}$ be the extension of $u_{j}$ to $\mathbb{R}^{n}$ by 0 outside $\Omega$. It is not difficult to see that $\tilde{u}_{j} \in$ $W^{1, q}\left(\mathbb{R}^{n} ; \mathbb{R}^{n}\right)$ and $\nabla \tilde{u}_{j}=\widetilde{\nabla u_{j}}$. Using $(2.10)$ with $D:=B(x, t)$ (so that $R=2 t$ ) and $\varphi:=\tilde{u}_{j}$ gives (recall that $t=|z|^{-\frac{1}{2}}$ )

$$
\begin{aligned}
t^{n\left(\frac{1}{q}-\frac{1}{q^{*}}\right)} & \left(\int_{B_{\Omega}(x, t)}\left|u_{j}\right|^{q^{*}} d y\right)^{\frac{1}{q^{*}}}=t^{n\left(\frac{1}{q}-\frac{1}{q^{*}}\right)}\left(\int_{B(x, t)}\left|\tilde{u}_{j}\right|^{q^{*}} d y\right)^{\frac{1}{q^{*}}} \\
& \leq C\left[\left(\int_{B(x, t)}\left|\tilde{u}_{j}\right|^{q} d y\right)^{\frac{1}{q}}+t\left(\int_{B(x, t)}\left|\nabla \tilde{u}_{j}\right|^{q} d y\right)^{\frac{1}{q}}\right] \\
& =C\left[\left(\int_{B_{\Omega}(x, t)}\left|u_{j}\right|^{q} d y\right)^{\frac{1}{q}}+t\left(\int_{B_{\Omega}(x, t)}\left|\nabla u_{j}\right|^{q} d y\right)^{\frac{1}{q}}\right] \\
& \leq C e^{-c 2^{j}} t^{n\left(\frac{1}{q}-\frac{1}{2}\right)} t^{2}\left(\int_{\Omega}\left|f_{j}\right|^{2} d y\right)^{\frac{1}{2}} .
\end{aligned}
$$


Therefore, (2.8) holds with $q^{*}$ instead of $q$. Let us now assume that $q^{*} \leq q_{0}$. It remains to prove (2.9) with $q^{*}$ instead of $q$. To this end, pick $\zeta \in \mathscr{C}_{c}^{\infty}(B(x, t) ; \mathbb{R})$ such that $0 \leq \zeta \leq 1, \zeta \equiv 1$ on $B(x, t / 2),\|\nabla \zeta\|_{\infty} \leq \frac{c}{t}$ and $\left\|\nabla^{2} \zeta\right\|_{\infty} \leq \frac{c}{t^{2}}$. For each $j \geq 1$ we then have $\zeta u_{j} \in W^{1, q}\left(\Omega ; \mathbb{R}^{n}\right) \cap H_{0}^{1}\left(\Omega ; \mathbb{R}^{n}\right)$ and

$$
\begin{aligned}
L_{D, 0}\left(\zeta u_{j}\right)= & \zeta L_{D} u_{j}-\mu\left(2(\nabla \zeta \cdot \nabla) u_{j}+(\Delta \zeta) u_{j}\right) \\
& -\mu^{\prime}\left((\nabla \zeta) \operatorname{div} u_{j}+\left(\nabla \zeta \cdot \partial_{k} u_{j}+u_{j} \cdot \nabla \partial_{k} \zeta\right)_{1 \leq k \leq n}\right) .
\end{aligned}
$$

In particular, this proves that $\zeta u_{j} \in D\left(L_{D}\right)$. Hence, since $\zeta f_{j}=0$, we have that

$$
z \zeta u_{j}+L_{D}\left(\zeta u_{j}\right)=\mathcal{O}\left(\left|\nabla \zeta \| \nabla u_{j}\right|+\left|\nabla^{2} \zeta\right|\left|u_{j}\right|\right), \quad j \geq 1 .
$$

In addition,

$$
z \zeta u_{0}+L_{D}\left(\zeta u_{0}\right)=\zeta f_{0}+\mathcal{O}\left(|\nabla \zeta|\left|\nabla u_{0}\right|+\left|\nabla^{2} \zeta\right|\left|u_{0}\right|\right) .
$$

As far as $L_{D}\left(\zeta u_{j}\right)$ is concerned, we have the following estimates

$$
\begin{aligned}
\left\|L_{D}\left(\zeta u_{j}\right)\right\|_{q} \leq & C|z|\|\zeta\|_{\infty}\left\|u_{j}\right\|_{L^{q}\left(B_{\Omega}(x, t) ; \mathbb{R}^{n}\right)} \\
& +C\|\nabla \zeta\|_{\infty}\left\|\nabla u_{j}\right\|_{L^{q}\left(B_{\Omega}(x, t) ; \mathbb{R}^{n}\right)} \\
& +C\left\|\nabla^{2} \zeta\right\|_{\infty}\left\|u_{j}\right\|_{L^{q}\left(B_{\Omega}(x, t) ; \mathbb{R}^{n}\right)},
\end{aligned}
$$

if $j \geq 1$, and

$$
\begin{aligned}
\left\|L_{D}\left(\zeta u_{0}\right)\right\|_{q} \leq & C\|\zeta\|_{\infty}\left(|z|\left\|u_{0}\right\|_{L^{q}\left(B_{\Omega}(x, t) ; \mathbb{R}^{n}\right)}+\left\|f_{0}\right\|_{2}\right) \\
& +C\|\nabla \zeta\|_{\infty}\left\|\nabla u_{0}\right\|_{L^{q}\left(B_{\Omega}(x, t) ; \mathbb{R}^{n}\right)} \\
& +C\left\|\nabla^{2} \zeta\right\|_{\infty}\left\|u_{0}\right\|_{L^{q}\left(B_{\Omega}(x, t) ; \mathbb{R}^{n}\right) .}
\end{aligned}
$$

Moreover, by the mapping properties of the Lamé operator (see Theorem 4.1 below), since $2<q^{*} \leq q_{0}$ and $W^{-1, q^{*}}(\Omega) \hookrightarrow L^{q}(\Omega)$, we have

$$
\left\|\nabla\left(\zeta u_{j}\right)\right\|_{q^{*}} \leq C\left\|L_{D}\left(\zeta u_{j}\right)\right\|_{q}
$$

and, consequently,

$$
\left\|\zeta \nabla u_{j}\right\|_{q^{*}} \leq C\left\|L_{D}\left(\zeta u_{j}\right)\right\|_{q}+\left\|\left|\nabla \zeta\left\|u_{j} \mid\right\|_{q} .\right.\right.
$$

Using (2.18) together with (2.15) or (2.16), and keeping in mind that $t=|z|^{-\frac{1}{2}}$, we obtain

$$
\sqrt{|z|}\left(\int_{B_{\Omega}\left(x, \frac{t}{2}\right)}\left|\nabla u_{j}\right|^{q^{*}} d y\right)^{\frac{1}{q^{*}}} \leq C t^{n\left(\frac{1}{q^{*}}-\frac{1}{2}\right)} e^{-c 2^{j}}\left\|f_{j}\right\|_{2} \quad \forall j \in \mathbb{N} .
$$

This is the version of (2.9) for $q^{*}$ instead of $q$, hence the proof of the lemma is complete.

After this preamble, we are ready to present the 
Proof of Proposition 2.1. The strategy is to first show that (2.8) and (2.9) hold for $q=2$ and then apply the iteration lemma (at most $\frac{n}{2}+1$ times), and interpolation. Turning to specifics, pick a family of functions $\left(\xi_{j}\right)_{j \geq 1}$ such that $\xi_{j} \in \mathscr{C}_{c}^{\infty}\left(B\left(x, 2^{j-1} t\right) ; \mathbb{R}\right)$. Taking the (complex) $L^{2}$-pairing of $\xi_{j}^{2} u_{j}$ with both sides of the equality $z u_{j}+L_{D} u_{j}=f_{j}$ yields

$$
\left\langle z u_{j}+L_{D} u_{j}, \xi_{j}^{2} u_{j}\right\rangle=\left\langle f_{j}, \xi_{j}^{2} u_{j}\right\rangle=0
$$

since $f_{j}$ and $\xi_{j}$ have disjoint supports for all $j \geq 1$. Denote by $m$ the minimum between $\mu$ and $\mu+\mu^{\prime}$, so that $m>0$ by the assumption (1.7). Based on repeated integration by parts we may then write

$$
\begin{aligned}
z \int_{\Omega} \xi_{j}^{2}\left|u_{j}\right|^{2} d y+ & m \int_{\Omega} \xi_{j}^{2}\left[\left|\operatorname{curl} u_{j}\right|^{2}+\left|\operatorname{div} u_{j}\right|^{2}\right] d y \\
& =\int_{\Omega} \mathcal{O}\left(\left|\nabla \xi_{j}\right|\left|u_{j}\right|\left|\xi_{j}\right|\left[\left|\operatorname{curl} u_{j}\right|+\left|\operatorname{div} u_{j}\right|\right]\right) d y
\end{aligned}
$$

From this, via Cauchy-Schwarz inequality used in the right hand-side of (2.21) for the functions $\left|\nabla \xi_{j}\right|\left|u_{j}\right|$ and $\xi_{j}\left[\left|\operatorname{curl} u_{j}\right|+\left|\operatorname{div} u_{j}\right|\right]$, we obtain

$$
\begin{aligned}
& z \int_{\Omega} \xi_{j}^{2}\left|u_{j}\right|^{2} d y+m \int_{\Omega} \xi_{j}^{2}\left[\left|\operatorname{curl} u_{j}\right|^{2}+\left|\operatorname{div} u_{j}\right|^{2}\right] d y \\
& \leq C_{0}\left(\int_{\Omega}\left|\nabla \xi_{j}\right|^{2}\left|u_{j}\right|^{2} d y\right)^{\frac{1}{2}}\left(\int_{\Omega} \xi_{j}^{2}\left[\left|\operatorname{curl} u_{j}\right|+\left|\operatorname{div} u_{j}\right|\right]^{2} d y\right)^{\frac{1}{2}}
\end{aligned}
$$

Recall the elementary inequalities

$$
\begin{aligned}
|a b| & \leq \frac{a^{2}}{2 \varepsilon}+\frac{\varepsilon b^{2}}{2} \\
a^{2}+b^{2} \leq(a+b)^{2} & \leq 2\left(a^{2}+b^{2}\right)
\end{aligned}
$$

valid for any numbers $a, b \in \mathbb{R}$, and $\varepsilon>0$. Applying first (2.23) to 2.22), then (2.24), with $a=\left|\operatorname{curl} u_{j}\right|$ and $b=\left|\operatorname{div} u_{j}\right|$, and choosing $\varepsilon=\frac{m}{c_{0}}$, allows us to absorb the second term of the sum on the left-hand side of (2.21). In this fashion we obtain

$$
|z| \int_{\Omega} \xi_{j}^{2}\left|u_{j}\right|^{2} d y \leq C \int_{\Omega}\left|\nabla \xi_{j}\right|^{2}\left|u_{j}\right|^{2} d y
$$

with $C=\frac{C_{0}^{2}}{2 m}$. The same type of procedure (changing the role of $u_{j}$ and $\left|\operatorname{curl} u_{j}\right|+\left|\operatorname{div} u_{j}\right|$ in the previous step), allows us to estimate

$$
\int_{\Omega} \xi_{j}^{2}\left[\left|\operatorname{curl} u_{j}\right|^{2}+\left|\operatorname{div} u_{j}\right|^{2}\right] d y
$$

in terms of

$$
\int_{\Omega}\left|\nabla \xi_{j}\right|^{2}\left[\left|\operatorname{curl} u_{j}\right|^{2}+\left|\operatorname{div} u_{j}\right|^{2}\right] d y .
$$

We therefore arrive at

$$
\int_{\Omega} \xi_{j}^{2}\left[\left|\operatorname{curl} u_{j}\right|^{2}+\left|\operatorname{div} u_{j}\right|^{2}\right] d y \leq \frac{C}{|z|} \int_{\Omega}\left|\nabla \xi_{j}\right|^{2}\left[\left|\operatorname{curl} u_{j}\right|^{2}+\left|\operatorname{div} u_{j}\right|^{2}\right] d y,
$$


for a finite constant $C>0$. To continue, let us now abbreviate

$$
w_{j}:=\left|\operatorname{curl} u_{j}\right|+\left|\operatorname{div} u_{j}\right| .
$$

Taking (2.24) into account, (2.28) becomes

$$
|z| \int_{\Omega} \xi_{j}^{2}\left|w_{j}\right|^{2} d y \leq C \int_{\Omega}\left|\nabla \xi_{j}\right|^{2}\left|w_{j}\right|^{2} d y .
$$

Much as in [2], we now replace the cutoff function $\xi_{j}$ by $e^{\alpha_{j} \xi_{j}}-1$ (which has the same properties as $\xi_{j}$ ), where $\alpha_{j}:=\frac{\sqrt{|z|}}{2 \sqrt{C}\left\|\nabla \xi_{j}\right\|_{\infty}}, j \geq 2$. In a first stage, this yields

$$
\int_{\Omega}\left|u_{j}\right|^{2}\left|e^{\alpha_{j} \xi_{j}}-1\right|^{2} d y \leq \frac{1}{4} \int_{\Omega}\left|u_{j}\right|^{2}\left|e^{\alpha_{j} \xi_{j}}\right|^{2} d y
$$

and

$$
\int_{\Omega}\left|w_{j}\right|^{2}\left|e^{\alpha_{j} \xi_{j}}-1\right|^{2} d y \leq \frac{1}{4} \int_{\Omega}\left|w_{j}\right|^{2}\left|e^{\alpha_{j} \xi_{j}}\right|^{2} d y
$$

then further

$$
\int_{\Omega}\left|u_{j}\right|^{2}\left|e^{\alpha_{j} \xi_{j}}\right|^{2} d y \leq 4 \int_{\Omega}\left|u_{j}\right|^{2} d y
$$

and

$$
\int_{\Omega}\left|w_{j}\right|^{2}\left|e^{\alpha_{j} \xi_{j}}\right|^{2} d y \leq 4 \int_{\Omega}\left|w_{j}\right|^{2} d y,
$$

in view of the generic, elementary implication

$$
\|f-g\| \leq \frac{1}{2}\|f\| \Longrightarrow\|f\| \leq 2\|g\| .
$$

If we now assume that the original cutoff functions $\left(\xi_{j}\right)_{j \geq 2}$ also satisfy $0 \leq \xi_{j} \leq$ $1, \xi_{j} \equiv 1$ on $B(x, t)$ and $\left\|\nabla \xi_{j}\right\|_{\infty} \leq \frac{\kappa}{2^{j} t}$, it follows from the definition of $\alpha_{j}$ that $\alpha_{j} \geq c 2^{j}$. Moreover, based on (2.33) and (2.34) we conclude that

$$
|z|\left|e^{\alpha_{j}}\right|^{2} \int_{B_{\Omega}(x, t)}\left|u_{j}\right|^{2} d y \leq 4 \int_{\Omega}\left|u_{j}\right|^{2} d y \leq C \int_{\Omega}\left|f_{j}\right|^{2} d y
$$

and

$$
\left|e^{\alpha_{j}}\right|^{2} \int_{B_{\Omega}(x, t)}\left|w_{j}\right|^{2} d y \leq 4 \int_{\Omega}\left|w_{j}\right|^{2} d y \leq \frac{C}{|z|} \int_{\Omega}\left|f_{j}\right|^{2} d y,
$$

the second inequalities being a consequence of the $L^{2}$-theory. This gives (2.8) for $q=2$ and also

$$
|z| \int_{B_{\Omega}(x, t)}\left[\left|\operatorname{curl} u_{j}\right|+\left|\operatorname{div} u_{j}\right|\right]^{2} d y \leq C e^{-c 2^{j}} \int_{\Omega}\left|f_{j}\right|^{2} d y .
$$

These two estimates are also valid if $j=0$ by the $L^{2}$-theory (if $j=0$, no decay is required in the estimates). We now claim that estimate (2.38) implies (2.9) 
for $q=2$. To justify this, let $\zeta \in \mathscr{C}_{c}^{\infty}(B(x, t) ; \mathbb{R})$ be such that $0 \leq \zeta \leq 1, \zeta \equiv 1$ on $B\left(x, \frac{t}{2}\right)$ and $\|\nabla \zeta\|_{\infty} \leq \frac{c}{t}$. In concert, the estimate (2.8) for $q=2$ and (2.38) imply that

$$
|z| \int_{\Omega}\left[\left|\operatorname{curl}\left(\zeta u_{j}\right)\right|^{2}+\left|\operatorname{div}\left(\zeta u_{j}\right)\right|^{2}\right] d y \leq C e^{-c 2^{j}}\left\|f_{j}\right\|_{2}^{2} .
$$

In addition, we also have $\zeta u_{j}=0$ on $\partial \Omega$. The inequality

$$
\|\Delta \varphi\|_{H^{-1}\left(\Omega ; \mathbb{R}^{n}\right)} \leq C\left(\|\operatorname{curl} \varphi\|_{2}+\|\operatorname{div} \varphi\|_{2}\right), \quad \varphi \in H_{0}^{1}\left(\Omega ; \mathbb{R}^{n}\right),
$$

implies in particular that $\Delta\left(\zeta u_{j}\right) \in H^{-1}\left(\Omega ; \mathbb{R}^{n}\right)$. Therefore, by the Lax-Milgram's theorem, we may conclude that $\zeta u_{j} \in H_{0}^{1}\left(\Omega ; \mathbb{R}^{n}\right)$ with norm bounded by a fixed multiple of $e^{-c 2^{j}}\left\|f_{j}\right\|_{2}$. This gives (2.9) for $q=2$. To obtain the estimates for $q>2$, we apply the iteration lemma (Lemma 2.2) which works as long as $\left(\ldots\left(\left(2^{*}\right)^{*}\right) \ldots\right)^{*} \leq q_{0}$. We then obtain $(2.8)$ and $(2.9)$ for all $q \in\left[2, q_{0}\right]$ by interpolation (between Lebesgue spaces), as well as (2.8) for all $q \in\left[q_{0}, q_{0}^{*}\right]$ by Sobolev's embedding (2.10) (using (2.8) and (2.9) for $q_{*} \in\left[\left(q_{0}\right)_{*}, q_{0}\right]$ ).

Having disposed of Proposition 2.1, we are now prepared to deal with the

Proof of Theorem 1.3. We proceed as in 19], Theorem 6.1. Consider first the case when $q \in\left(2, q_{0}^{*}\right]$. In this scenario, for an arbitrary $z \in \Sigma_{\theta}$, the fact that the operator $z I+L_{D}^{q}$ is one-to-one follows trivially from the corresponding statement for $q=2$. To see that this operator is also onto, let $f \in L^{q}\left(\Omega ; \mathbb{R}^{n}\right) \subset L^{2}\left(\Omega ; \mathbb{R}^{n}\right)$ and consider $u:=\left(z I+L_{D}\right)^{-1} f \in D\left(L_{D}\right)$. Then, as shown below, $u \in L^{q}\left(\Omega ; \mathbb{R}^{n}\right)$ and then, since $L_{D} u=f-z u \in L^{q}\left(\Omega ; \mathbb{R}^{n}\right)$, this proves that $u \in D\left(L_{D}^{p}\right)$ and $z u+L_{D}^{q} u=f$, which proves that $L_{D}^{q}$ is indeed onto. Let us now prove that $u \in L^{q}\left(\Omega ; \mathbb{R}^{n}\right)$. To do that, consider first smooth vector fields $f \in \mathscr{C}_{c}^{\infty}\left(\Omega ; \mathbb{R}^{n}\right)$. Applying the same procedure as before, we may write $f=\sum_{j=0}^{\infty} f_{j}, u=\sum_{j=0}^{\infty} u_{j}$ such that (2.8) holds.

To proceed, given a measurable subset $E$ of $\mathbb{R}^{n}$ with $|E|>0$ and an integrable function $g: E \rightarrow \mathbb{C}$ we set

$$
f_{E} g d x:=\frac{1}{|E|} \int_{E} g d x
$$

Recall that $t=|z|^{-\frac{1}{2}}$ and that $M_{\Omega}$ denotes the Hardy-Littlewood maximal 
operator in $\Omega$. Fubini's theorem then allows us to write

$$
\begin{aligned}
& |z|\left[\int_{\Omega}|u|^{q} d x\right]^{\frac{1}{q}} \leq C|z|\left[\int_{\Omega}\left(f_{B_{\Omega}(x, t)}|u|^{q} d y\right) d x\right]^{\frac{1}{q}} \\
& =C|z|\left\{\int_{\Omega}\left[\left(f_{B_{\Omega}(x, t)}|u|^{q} d y\right)^{\frac{1}{q}}\right]^{q} d x\right\}^{\frac{1}{q}} \\
& \leq C|z|\left\{\int_{\Omega}\left[\left(\sum_{j=0}^{\infty} f_{B_{\Omega}(x, t)}\left|u_{j}\right|^{q} d y\right)^{\frac{1}{q}}\right]^{q} d x\right\}^{\frac{1}{q}} \\
& \leq C\left\{\int_{\Omega}\left[\sum_{j=0}^{\infty} C e^{-c 2^{j}} 2^{j \frac{n}{2}}\left(f_{B_{\Omega}\left(x, 2^{j+1} t\right)}|f|^{2} d y\right)^{\frac{1}{2}}\right]^{q} d x\right\}^{\frac{1}{q}} \\
& \leq C\left(\sum_{j=0}^{\infty} C e^{-c 2^{j}} 2^{j \frac{n}{2}}\right)\left(\int_{\Omega} M_{\Omega}\left(|f|^{2}\right)^{\frac{q}{2}} d x\right)^{\frac{1}{q}} \\
& \leq C\left\|M_{\Omega}\left(|f|^{2}\right)\right\|_{\frac{q}{2}}^{\frac{1}{2}} \leq C\|f\|_{q} .
\end{aligned}
$$

The third inequality is obtained by writing $u=\sum_{j=0}^{\infty} u_{j}$. The fourth inequality is a consequence of (2.8) for $j \in \mathbb{N}$. The fifth inequality is implied by (1.14), which also yields the last inequality. Since $\mathscr{C}_{c}^{\infty}\left(\Omega ; \mathbb{R}^{n}\right)$ is dense in $L^{q}\left(\Omega ; \mathbb{R}^{n}\right)$, this proves that for all $q \in\left(2, q_{0}^{*}\right]$ and all $\theta \in(0, \pi)$ there exists a constant $C>0$ such that for all functions $f \in L^{q}\left(\Omega ; \mathbb{R}^{n}\right)$ and all numbers $z \in \Sigma_{\theta}$, the solution $u$ of $z u+L_{D} u=f$ satisfies

$$
|z|\|u\|_{q} \leq C\|f\|_{q}
$$

This implies that $-L_{D}^{q}$ generates an analytic semigroup in $L^{q}\left(\Omega ; \mathbb{R}^{n}\right)$ for all $q \in\left[2, q_{0}^{*}\right]$ (the case $q=2$ is covered by the discussion in last part of $\S 1.1$ ). Moreover, since $L_{D}$ is self-adjoint, by duality this gives that $-L_{D}^{q}$ generates an analytic semigroup in $L^{q}\left(\Omega ; \mathbb{R}^{n}\right)$ for all $q \in\left[\left(q_{0}^{*}\right)^{\prime}, 2\right]$ as well.

Finally, we now turn to the

Proof of Theorem 1.4. This is a direct consequence of estimate 2.8) by applying Theorem 2.2 and Remark 7.2 of [13]. Indeed, as already mentioned at the beginning of this section, $\Omega$ equipped with the standard Euclidean distance and the Lebesgue measure induced by $\mathbb{R}^{n}$ is a space of homogeneous type, in which scenario the results of 13 (see also [3]) apply.

\section{A discussion from the perspective of general second-order elliptic systems}

In this section we elaborate on the nature of the approach pursued in $\S 2$. We start from the observation that there are infinitely many ways of writing the 
Lamé system in the form of a general elliptic second order partial differential operator. More specifically, for a fixed, arbitrary parameter $r \in \mathbb{R}$, set (using the standard $\delta$-Kronecker formalism)

$$
a_{\ell k}^{\alpha \beta}(r):=\mu \delta_{\ell k} \delta_{\alpha \beta}+\left(\mu^{\prime}-r\right) \delta_{\ell \alpha} \delta_{k \beta}+r \delta_{\ell \beta} \delta_{k \alpha} .
$$

Then for any vector field $u=\left(u_{\alpha}\right)_{1 \leq \alpha \leq n}$ and any $\alpha=1, \ldots, n$ we have (using the repeated index summation convention)

$$
-\partial_{\ell}\left(a_{\ell k}^{\alpha \beta}(r) \partial_{k} u_{\beta}\right)=-\mu \Delta u_{\alpha}-\mu^{\prime} \partial_{\alpha}(\operatorname{div} u)
$$

i.e., the $\alpha$-component of the Lamé operator $-\mu \Delta-\mu^{\prime} \nabla$ div acting on $u$.

The bilinear form associated with the representation of the Lamé operator as in (3.1)-3.2) in an open subset $\Omega$ of $\mathbb{R}^{n}$ is

$$
Q_{r}(u, v):=\int_{\Omega} a_{\ell k}^{\alpha \beta}(r) \partial_{k} u_{\beta} \overline{\partial_{\ell} v_{\alpha}} d x, \quad u, v \in H_{0}^{1}\left(\Omega ; \mathbb{R}^{n}\right) .
$$

To be more pedantic, we should write $Q_{\mu, \mu^{\prime}, r}$ but we will only do so whenever the circumstances dictate it. In general, let $A=\left(a_{\ell k}^{\alpha \beta}\right)_{\substack{1 \leq \ell, k \leq n \\ 1 \leq \alpha, \beta \leq m}}$ be a coefficient tensor to which we associate the second order constant coefficients operator $L$ in $\mathbb{R}^{n}$ as follows

$$
L u:=-\left(a_{\ell k}^{\alpha \beta} \partial_{\ell} \partial_{k} u_{\beta}\right)_{1 \leq \alpha \leq m} .
$$

Two different coefficient tensors $A$ may have the same associated operator $L$, as is apparent by writing the Lamé operator in the form (3.1)-(3.2) (if necessary, we may use the symbol $L_{A}$ to indicate what tensor coefficient $A$ has been used in the writing of the operator $L$ ).

For an operator as in (3.4), the Legendre-Hadamard elliptic condition reads

$$
\Re e\left(a_{\ell k}^{\alpha \beta} \xi_{\ell} \xi_{k} \eta_{\alpha} \overline{\eta_{\beta}}\right) \geq \kappa|\xi|^{2}|\eta|^{2}, \quad \forall \xi \in \mathbb{R}^{n}, \forall \eta \in \mathbb{C}^{m},
$$

for a positive constant $\kappa$. To a coefficient tensor $A=\left(a_{\ell k}^{\alpha \beta}\right)_{\substack{1 \leq \ell, k \leq n \\ 1 \leq \alpha, \beta \leq m}}$ we associate the quadratic form

$$
Q_{A}(u, v):=\int_{\Omega} a_{\ell k}^{\alpha \beta} \partial_{\ell} u_{\alpha} \overline{\partial_{k} v_{\beta}} d x \quad u, v \in H_{0}^{1}\left(\Omega ; \mathbb{C}^{m}\right) .
$$

Note that if $A$ satisfies (3.5), then $Q_{A}$ is coercive on $H_{0}^{1}\left(\Omega ; \mathbb{C}^{m}\right)$. Indeed an application of Plancherel theorem along with the observation that $u \in H_{0}^{1}\left(\Omega ; \mathbb{C}^{m}\right)$ entails $\tilde{u} \in H_{0}^{1}\left(\mathbb{R}^{n} ; \mathbb{C}^{m}\right)$ and $\nabla \tilde{u}=\widetilde{\nabla u}$ (where $\tilde{u}$ denotes the extension of $u$ by 0 outside $\Omega$ ) allow us to estimate

$$
\begin{aligned}
Q_{A}(u, u) & =\int_{\mathbb{R}^{n}} a_{\ell k}^{\alpha \beta} \partial_{\ell} \tilde{u}_{\alpha} \overline{\partial_{k} \tilde{u}_{\beta}} d x=\int_{\mathbb{R}^{n}} a_{\ell k}^{\alpha \beta}\left(i \xi_{\ell} \widehat{\tilde{u}}_{\alpha}\right)\left(\overline{i \xi_{k} \widehat{\tilde{u}}_{\beta}}\right) d \xi \\
& =\int_{\mathbb{R}^{n}} a_{\ell k}^{\alpha \beta} \xi_{\ell} \xi_{k} \widehat{\tilde{u}}_{\alpha} \overline{\tilde{\tilde{u}}_{\beta}} d \xi \geq \kappa \int_{\mathbb{R}^{n}}|\xi|^{2}|\widehat{\tilde{u}}|^{2} d \xi \\
& \geq \kappa \int_{\mathbb{R}^{n}}|\nabla \tilde{u}|^{2} d x=\kappa \int_{\Omega}|\nabla u|^{2} d x
\end{aligned}
$$

where $\hat{\varphi}$ denotes the Fourier transform of a square-integrable function $\varphi$ defined on $\mathbb{R}^{n}$. 
Lemma 3.1. In the case of the Lamé operator, the coefficient tensor $A_{r}=$ $\left(a_{\ell k}^{\alpha \beta}(r)\right)_{1 \leq \ell, k \leq n}$ as defined in (3.1) satisfies the Legendre-Hadamard ellipticity condition (3.5) if and only if $\mu>0$ and $\mu+\mu^{\prime}>0$.

Proof. It is easy to see that the condition (3.5) for $A_{r}=\left(a_{\ell k}^{\alpha \beta}(r)\right)_{\substack{1 \leq \ell, k \leq n \\ 1 \leq \alpha, \beta \leq m}}$ is equivalent to the existence of a constant $c>0$ such that

$$
\mu|\xi|^{2}|\eta|^{2}+\mu^{\prime}\langle\xi, \eta\rangle^{2} \geq c|\xi|^{2}|\eta|^{2}, \quad \xi, \eta \in \mathbb{R}^{n},
$$

where $\langle\cdot, \cdot\rangle$ denotes the standard Euclidean scalar product of vectors in $\mathbb{R}^{n}$. If $\xi=0$ or $\eta=0$, (3.8) is clearly verified. If $\xi \neq 0$ and $\eta \neq 0$, we may renormalize the vectors involved and assume that $|\xi|=|\eta|=1$. With $t:=\langle\xi, \eta\rangle^{2}$, the condition (3.8) reads then

$$
\mu+\mu^{\prime} t \geq c, \quad \text { for all } t \in[0,1] .
$$

Therefore, (3.8) is equivalent to

$$
\min \left\{\mu, \mu+\mu^{\prime}\right\}=\inf _{t \in[0,1]}\left(\mu+\mu^{\prime} t\right)>0,
$$

which proves the claim. Incidentally, the above reasoning also shows that the best constant $c$ in (3.8) is $\min \left\{\mu, \mu+\mu^{\prime}\right\}$.

Hence, the form $Q_{r}=Q_{r, \mu, \mu^{\prime}}$ introduced in (3.3) is coercive for all $r \in \mathbb{R}$ provided $\mu>0$ and $\mu+\mu^{\prime}>0$.

Proposition 3.2. The sesquilinear form $a_{D}$ defined in (1.6) used in Section 目 corresponds to $Q_{r, \mu, \mu^{\prime}}$ with $r=-\mu$. In other words, $a_{D}=Q_{-\mu}$.

Proof. By the definition (1.2) of the curl in $\mathbb{R}^{n}$, we have (using the repeated index summation convention)

$$
\begin{aligned}
\operatorname{curl} u \cdot \overline{\operatorname{curl} v}= & \frac{1}{2} \partial_{\ell} u_{\alpha} \overline{\partial_{k} v_{\beta}}\left(\left(\delta_{m \ell} \delta_{\gamma \alpha}-\delta_{\gamma \ell} \delta_{m \alpha}\right)\left(\delta_{m k} \delta_{\gamma \beta}-\delta_{\gamma k} \delta_{m \beta}\right)\right) \\
= & \frac{1}{2} \partial_{\ell} u_{\alpha} \overline{\partial_{k} v_{\beta}}\left(\delta_{k m} \delta_{m \ell} \delta_{\alpha \gamma} \delta_{\gamma \beta}-\delta_{\ell m} \delta_{m \beta} \delta_{\alpha \gamma} \delta_{\gamma k}\right. \\
& \left.-\delta_{\ell \gamma} \delta_{\gamma \beta} \delta_{\alpha m} \delta_{m k}+\delta_{\ell \gamma} \delta_{\gamma k} \delta_{\alpha m} \delta_{m \beta}\right) \\
= & \partial_{\ell} u_{\alpha} \overline{\partial_{k} v_{\beta}}\left(\delta_{\ell k} \delta_{\alpha \beta}-\delta_{\ell \beta} \delta_{k \alpha}\right) .
\end{aligned}
$$

Moreover, we have (with the same convention)

$$
\operatorname{div} u \overline{\operatorname{div} v}=\delta_{\ell \alpha} \delta_{k \beta} \partial_{\ell} u_{\alpha} \overline{\partial_{k} v_{\beta}} .
$$

Therefore, by the definition (1.6) of $a_{D}$, we have

$$
a_{D}(u, v)=\int_{\Omega} \partial_{\ell} u_{\alpha} \overline{\partial_{k} v_{\beta}}\left(\mu\left(\delta_{\ell k} \delta_{\alpha \beta}-\delta_{\ell \beta} \delta_{k \alpha}\right)+\left(\mu+\mu^{\prime}\right) \delta_{\ell \alpha} \delta_{k \beta}\right) d x
$$

and the coefficient $\mu \delta_{\ell k} \delta_{\alpha \beta}-\mu \delta_{\ell \beta} \delta_{k \alpha}+\left(\mu+\mu^{\prime}\right) \delta_{\ell \alpha} \delta_{k \beta}$ is of the form $a_{\ell k}^{\alpha \beta}(r)$ (defined in (3.1)) with $r=-\mu$. Therefore, $a_{D}=Q_{-\mu}$, as claimed. 
For certain applications it is important to know whether the coefficient tensor of a general operator $L$ (defined in (3.4)) satisfies a stronger ellipticity condition to the effect that

$$
\Re e\left(a_{\ell k}^{\alpha \beta} \zeta_{\ell}^{\alpha} \overline{\zeta_{k}^{\beta}}\right) \geq \kappa|\zeta|^{2}, \quad \forall \zeta=\left(\zeta_{\ell}^{\alpha}\right)_{\substack{1 \leq \ell \leq n \\ 1 \leq \alpha \leq m}} \in \mathbb{C}^{n \times m},
$$

for some $\kappa>0$. Note that (3.5) is implied by (3.14) (take $\left.\zeta_{\ell}^{\alpha}=\xi_{\ell} \eta_{\alpha}\right)$.

Proposition 3.3. In the case of the Lamé operator, (3.14) is satisfied for some $r \in \mathbb{R}$ if and only if $\mu>0$ and $\mu+\mu^{\prime}>0$. In this case, $|r|<\mu$.

Proof. The proof of this proposition follows from Lemma 3.4 below, which appears in [20, Lemma 4.1]. Indeed,

$$
\Re e\left(a_{\ell k}^{\alpha \beta}(r) \zeta_{\ell}^{\alpha} \overline{\zeta_{k}^{\beta}}\right)=\mathcal{Q}_{a, b, c}(\zeta) \quad \forall \zeta=\left(\zeta_{\ell}^{\alpha}\right)_{\substack{1 \leq \ell \leq n \\ 1 \leq \alpha \leq m}} \in \mathbb{C}^{n \times n}
$$

where $\mathcal{Q}_{a, b, c}$ is as in (3.16) with $a=\mu-r, b=2 r$ and $c=\mu^{\prime}-r$. From (3.17) it follows that (3.14) holds for $A_{r}$ if and only if $a>0, a+b>0$ and $a+b+c n>0$. Collectively, the conditions $a>0$ and $a+b>0$ are equivalent to the fact that $\mu>0$ and $-\mu<r<\mu$. The condition $a+b+c n>0$ is equivalent to $r>-\frac{\mu+n \mu^{\prime}}{n-1}$. There remains to observe that the intervals $(-\mu, \mu)$ and $\left(-\frac{\mu+n \mu^{\prime}}{n-1},+\infty\right)$ have a nonempty intersection if and only if $\mu>-\frac{\mu+n \mu^{\prime}}{n-1}$, i.e. if $\mu+\mu^{\prime}>0$.

Here is the algebraic result invoked above.

Lemma 3.4. For $\zeta=\left(\zeta_{j}^{\alpha}\right)_{\alpha, j}$ an $n \times n$ matrix, $n \geq 2$, and $a, b, c \in \mathbb{R}$, let

$$
\mathcal{Q}(\zeta)=\mathcal{Q}_{a, b, c}(\zeta):=a|\zeta|^{2}+b\left|\frac{1}{2}\left(\zeta+\zeta^{\top}\right)\right|^{2}+c|\operatorname{Tr}(\zeta)|^{2}
$$

where $\operatorname{Tr}$ stands for the usual matrix-trace operator, $\top$ denotes transposition of matrices, and we have set $|\zeta|:=\left[\operatorname{Tr}\left(\zeta \zeta^{\top}\right)\right]^{1 / 2}$. Then

$$
\exists \kappa>0 \text { with } \mathcal{Q}(\zeta) \geq \kappa|\zeta|^{2} \quad \forall \zeta \Longleftrightarrow\left\{\begin{array}{l}
a>0, \\
a+b>0, \\
a+b+c n>0 .
\end{array}\right.
$$

and

$$
\begin{aligned}
& \text { there exists } \kappa>0 \text { such that for every matrix } \zeta \text { one has } \\
& \mathcal{Q}(\zeta) \geq \kappa\left(\left|\frac{1}{2}\left(\zeta-\zeta^{\top}\right)\right|^{2}+|\operatorname{Tr} \zeta|^{2}\right) \Longleftrightarrow\left\{\begin{array}{l}
a>0, \\
a+b \geq 0, \\
a+b+c n>0 .
\end{array}\right.
\end{aligned}
$$

We would now like to comment on the significance of Proposition 3.3 in the context of the estimates carried out in Section 2. Specifically, granted (3.14) and the symmetry condition

$$
a_{\ell k}^{\alpha \beta}=\overline{a_{k \ell}^{\beta \alpha}}, \quad \text { whenever } 1 \leq \alpha, \beta \leq m, 1 \leq \ell, k \leq n
$$


it follows that there exists a coefficient tensor $S=\left(b_{\ell k}^{\alpha \beta}\right)_{\substack{1 \leq \ell, k \leq n \\ 1 \leq \alpha, \beta \leq m}}$ with complex entries which is symmetric (i.e. the coefficients $b_{\ell k}^{\alpha \beta}$ satisfy (3.19)), and such that

$$
\left\langle A \zeta, \zeta^{\prime}\right\rangle=\left\langle S \zeta, S \zeta^{\prime}\right\rangle, \quad \forall \zeta, \zeta^{\prime} \in \mathbb{C}^{n \times m} .
$$

Furthermore, $S$ is bounded from below by $\kappa^{\frac{1}{2}}$, i.e. in operator theoretic sense,

$$
S \geq \kappa^{\frac{1}{2}} I_{\mathbb{C}^{n \times m}}
$$

Having $S$ as in (3.20) and (3.21) allows one to carry out the program developed in Section 2 for any operator $L_{A}$ with $A$ as in (3.14) and (3.19). Indeed, the analogue of (2.21) is

$$
z \int_{\Omega} \xi_{j}^{2}\left|u_{j}\right|^{2} d x+\int_{\Omega} \xi_{j}^{2}\left|S \nabla u_{j}\right|^{2} d x=\int_{\Omega} \mathcal{O}\left(\left|\nabla \xi_{j}\right|\left|\xi_{j}\right|\left|u_{j}\right|\left|S \nabla u_{j}\right|\right) d x
$$

We stress that this structure is crucial for the ability to estimate

$$
\int_{\Omega} \xi_{j}^{2}\left|S \nabla u_{j}\right|^{2} d x \leq \frac{C}{|z|} \int_{\Omega}\left|\nabla \xi_{j}\right|^{2}\left|S \nabla u_{j}\right|^{2} d x
$$

in place of (2.28) (the proof of (2.25) is similar as before, as this part is not sensitive to the underlying algebraic structure). With this in hand, the proof then proceeds as before, this time setting $w_{j}:=S \nabla u_{j}$ in place of (2.29). As a result, we arrive at

$$
|z| \int_{B_{\Omega}(x, t)}\left|S \nabla u_{j}\right|^{2} d x \leq C e^{-c 2^{j}}\left\|f_{j}\right\|_{2}^{2}
$$

in place of (2.38). At this stage it is no longer necessary to use the cutoff function $\zeta$ as done in (2.38), the reason being the fact that $S$ is coercive. Hence, we can directly estimate

$$
\int_{B_{\Omega}(x, t)}\left|\nabla u_{j}\right|^{2} d x \leq C \int_{B_{\Omega}(x, t)}\left|S \nabla u_{j}\right|^{2} d x
$$

and eventually obtain $(2.9)$ for $q=2$.

In conjunction with the above discussion, two relevant points are as follows. First, it is possible to prove $L^{p}$-maximal regularity in $L^{q}\left(\Omega ; \mathbb{R}^{n}\right)$ for a general system $L_{A}$ as in (3.4) satisfying (3.14) and (3.19), at least for $q \in\left(\left(2^{*}\right)^{\prime}, 2^{*}\right)$. Further regularity results can, when available, be used to extend this to a larger interval. Second, the Lamé system is amenable to this type of treatment. Indeed, by Proposition 3.3 , there exists $r \in \mathbb{R}$ such that $A_{r}=\left(a_{\ell k}^{\alpha \beta}(r)\right)_{\substack{1 \leq \ell, k \leq n \\ 1 \leq \alpha, \beta \leq n}}$ is strongly elliptic in the sense of (3.14), provided $\mu>0$ and $\mu+\mu^{\prime}>0$.

Intriguingly enough, our treatment of Lamé from Section 2 did not proceed according to the scheme outlined above, even though the quadratic form that we use was of type $Q_{r}$ (with $r=-\mu$, cf. Proposition 3.2). Indeed, the choice $r=-\mu$ yields a coefficient tensor $A_{r}$ which is not strongly elliptic (cf. Proposition 3.3). However, by Lemma 3.4, (3.18), $Q_{r}$ with $r=-\mu$ does satisfy the weaker coercivity condition

$$
Q_{r}(u, u) \geq \kappa\left(\| \frac{1}{2}\left(\nabla u-(\nabla u)^{\top}\left\|_{2}^{2}+\right\| \operatorname{Tr} \nabla u \|_{2}^{2}\right), \quad \forall u, v \in H_{0}^{1}\left(\Omega ; \mathbb{R}^{n}\right) .\right.
$$


Indeed, for $a=\mu-r, b=r$ and $c=\mu^{\prime}-r$, (3.18) gives the estimate (3.26), granted that $\mu>0, \mu+\mu^{\prime}>0$ and $|r| \leq \mu$. Accordingly, by (3.18), the coefficient tensor $A_{-\mu}$ has a square root $S$ in the sense of (3.20) which this time only satisfies

$$
|S \zeta| \geq c\left(\left|\zeta-\zeta^{\top}\right|+|\operatorname{Tr} \zeta|\right), \quad \forall \zeta \in \mathbb{C}^{n \times n}
$$

for a positive constant $c$. As a result, since $\operatorname{Tr} \nabla u=\operatorname{div} u$ and $|\operatorname{curl} u| \approx \mid \nabla u-$ $(\nabla u)^{\top} \mid$ (cf. $\left.(1.2)\right)$, instead of estimating $|S \nabla u|$ with $|\nabla u|$ from below, this time we only have (compare with (2.38)

$$
|S \nabla u| \geq c(|\operatorname{curl} u|+|\operatorname{div} u|) \quad \forall u \in H_{0}^{1}\left(\Omega ; \mathbb{R}^{n}\right) .
$$

Nevertheless, it is the fortunate fact that the div-curl system is elliptic which eventually allows us (via PDE methods, and not just abstract functional analysis results) to conclude that, even in this special critical case, we still have

$$
\int_{B_{\Omega}(x, t)}|S \nabla u|^{2} d y \geq c \int_{B_{\Omega}\left(x, \frac{t}{2}\right)}|\nabla u|^{2} d y, \quad \forall u \in H_{0}^{1}\left(\Omega ; \mathbb{R}^{n}\right) .
$$

In particular, this explains the necessity of invoking the elliptic estimate (2.40).

\section{Regularity results}

Given a reasonable domain $\Omega \subset \mathbb{R}^{n}$, denote by $\operatorname{VMO}(\partial \Omega)$ the Sarason space of functions of vanishing mean oscillations on $\partial \Omega$ (defined, e.g., as the closure of the space of Lipschitz functions on $\partial \Omega$ in the John-Nirenberg space $\mathrm{BMO}(\partial \Omega)$ of functions of bounded mean oscillations). Recall that the family of $(\varepsilon, \delta)$ domains, introduced by P. Jones in [10], is the largest class of Sobolev extension domains currently known (in fact, in the two dimensional setting this class is optimal; see 10] for definitions and other pertinent comments). The regularity result used in the previous sections of the paper reads as follows.

Theorem 4.1. Let $\Omega \subset \mathbb{R}^{n}$ be a bounded open set and, for $q \in(1, \infty)$, consider the following Poisson problem for the Lamé system

$$
u \in W_{0}^{1, q}\left(\Omega ; \mathbb{R}^{n}\right), \quad \mu \Delta u+\mu^{\prime} \nabla \operatorname{div} u=f \in W^{-1, q}\left(\Omega ; \mathbb{R}^{n}\right) \quad \text { in } \Omega .
$$

The following situations describe contexts in which the problem (4.1) is wellposed:

(i) $\mu>0, \mu+\mu^{\prime}>0, q \in(1, \infty)$ and $\Omega$ is bounded an $(\varepsilon, \delta)$-domain with an Ahlfors-David regular boundary and whose outward unit normal $\nu$ has the property that (with the distance taken in $\mathrm{BMO}(\partial \Omega)$ )

$$
\operatorname{dist}(\nu, \operatorname{VMO}(\partial \Omega))<\eta,
$$

where $\eta>0$ is a small number which depends exclusively on $q$ and the aforementioned geometrical characteristics of $\Omega$.

(ii) $\mu>0, \mu+\mu^{\prime}>0, q \in(1, \infty)$ and $\Omega$ is bounded an $(\varepsilon, \delta)$-domain with an Ahlfors-David regular boundary and such that

$$
\nu \in \operatorname{VMO}(\partial \Omega) .
$$


(iii) $\mu>0, \mu+\mu^{\prime}>0, \Omega$ is an arbitrary bounded Lipschitz domain, and

$$
q \in\left(\left(q_{\Omega}\right)^{\prime}, q_{\Omega}\right), \quad \text { where } q_{\Omega}:=\frac{2 n}{n-1-\varepsilon(n-1)},
$$

where $\varepsilon \in(0,1]$ depends only on the Lipschitz character of $\Omega$.

(iv) $\mu>0, \mu+\mu^{\prime}>0, q \in(1, \infty)$ arbitrary and $\Omega$ is a bounded $\mathscr{C}^{1}$ domain (or, more generally, a bounded Lipschitz domain with the property that (4.3) holds).

This is a collection of results proved elsewhere (items (i)-(ii) are implied by more general results proved in [18], item (iii) is proved in 23] and 21] (cf. also [17. for the lower dimensional case), while item (iv) is a particular case of (i)), and here we only wish to elaborate on the terminology and outline the strategy used in [18] to prove (ii).

We begin by recording a theorem, itself a particular case of a more general result from [8], whose relevance for the current discussion will become apparent shortly. To state it, denote by $\mathcal{L}(\mathcal{X})$ the Banach space of bounded linear operators from the Banach space $\mathcal{X}$ into itself, and by $\operatorname{Comp}(\mathcal{X})$ the closed two-sided ideal consisting of compact mappings of $\mathcal{X}$ into itself.

Theorem 4.2. Let $\Omega$ be an open set in $\mathbb{R}^{n}$ satisfying a two-sided local John condition and whose boundary is Ahlfors-David regular and compact. Denote by $\sigma$ the surface measure on $\partial \Omega$ and by $\nu$ the outward unit normal to $\Omega$. Also, fix a function $k \in C^{\infty}\left(\mathbb{R}^{n} \backslash\{0\}\right)$ which is even and homogeneous of degree $-n$, and set

$$
T f(x):=\lim _{\varepsilon \rightarrow 0} \int_{\substack{y \in \partial \Omega \\|x-y|>\varepsilon}}\langle x-y, \nu(y)\rangle k(x-y) f(y) d \sigma(y), \quad x \in \partial \Omega .
$$

Then for every $q \in(1, \infty)$ the following implication is valid:

$\nu \in \mathrm{VMO}(\partial \Omega) \Longrightarrow T: L^{q}(\partial \Omega) \longrightarrow L^{q}(\partial \Omega)$ is a compact operator.

For precise definitions, the interested reader is referred to [8] and the references therein. Here it suffices to say that the surface measure $\sigma$ is the restriction of the $(n-1)$-dimensional Hausdorff measure to $\partial \Omega$, and the unit normal is taken in the sense of De Georgi-Federer (given that $\Omega$ is a set of locally finite perimeter). Also, the Ahlfors-David regularity condition refers to the fact that $\partial \Omega$ behaves, from the point of view of measure theory, like a $(n-1)$-dimensional surface (in fact, this is not a regularity statement per se, but rather a demand that the measure of any surface ball of radius $r$ behaves like $r^{n-1}$ at all scales and locations). Finally, the two-sided local John condition is a quantitative, scale invariant weak form of non-tangential accessibility of boundary points, both from inside and outside of $\Omega$.

The conormal derivative associated with the above choice of coefficients in the writing of the Lamé operator as in (3.1)-(3.2), is given by

$$
\partial_{\nu}^{r} u:=\left(\nu_{j} a_{j k}^{\alpha \beta}(r) \partial_{k} u_{\beta}\right)_{\alpha}=\left.\left[\mu(\nabla u)^{\top}+r(\nabla u)\right]\right|_{\partial \Omega} \nu+\left.\left(\mu^{\prime}-r\right)(\operatorname{div} u)\right|_{\partial \Omega} \nu,
$$


where the superscript $T$ denotes transposition. The approach to solving the Dirichlet problem

$$
\mu \Delta u+\mu^{\prime} \nabla \operatorname{div} u=0 \text { in } \Omega, \quad u=g \text { on } \partial \Omega,
$$

via the method of boundary integral operators proceeds as follows. Let $\omega_{n-1}$ denote the surface measure of the unit sphere in $\mathbb{R}^{n}$, and let $E(x)=\left(E_{j k}(x)\right)_{1 \leq j, k \leq n}$ be the standard fundamental solution for the Lamé system, defined at each $x=\left(x_{j}\right)_{j} \in \mathbb{R}^{n} \backslash\{0\}$ by

$$
E_{j k}(x):= \begin{cases}\frac{-1}{2 \mu\left(\mu+\mu^{\prime}\right) \omega_{n-1}}\left[\frac{2 \mu+\mu^{\prime}}{n-2} \frac{\delta_{j k}}{|x|^{n-2}}+\mu^{\prime} \frac{x_{j} x_{k}}{|x|^{n}}\right], \quad \text { if } n \geq 3, \\ \frac{1}{2 \pi \mu\left(\mu+\mu^{\prime}\right)}\left[\left(2 \mu+\mu^{\prime}\right) \delta_{j k} \log |x|-\mu^{\prime} \frac{x_{j} x_{k}}{|x|^{2}}\right], & \text { if } n=2 .\end{cases}
$$

See, e.g., [16] and (9.2) in Chapter 9 of $[15]$. For each $r \in \mathbb{R}$, we then define the elastic double layer potential operator $\mathcal{D}_{r}$ acting on a vector field $h$ on $\partial \Omega$ by setting

$$
\mathcal{D}_{r} h(x):=\int_{\partial \Omega}\left[\partial_{\nu(y)}^{r} E(y-x)\right]^{\top} h(y) d \sigma(y), \quad x \in \Omega .
$$

For a reasonable domain $\Omega \subset \mathbb{R}^{n}$ we seek a solution to (4.8) in the form $u:=\mathcal{D}_{r} h$ for a suitable vector field $h$ on $\partial \Omega$, in which case it is useful to know that

$$
\left.\mathcal{D}_{r} h\right|_{\partial \Omega}=\left(\frac{1}{2} I+K_{r}\right) h,
$$

where $I$ denotes the identity operator, the restriction to the boundary is taken in a pointwise non-tangential sense, and

$$
K_{r} h(x):=\lim _{\varepsilon \rightarrow 0^{+}} \int_{\substack{y \in \partial \Omega \\|x-y|>\varepsilon}}\left[\partial_{\nu(y)}^{r} E(y-x)\right]^{\top} h(y) d \sigma(y), \quad x \in \partial \Omega .
$$

Explicitly, the integral kernel of the operator 4.12 is a $n \times n$ matrix whose $(j, k)$ entry is given by

$$
\begin{aligned}
& -L_{1}(r) \frac{\delta_{j k}}{\omega_{n-1}} \frac{\langle x-y, \nu(y)\rangle}{|x-y|^{n}}-\left(1-L_{1}(r)\right) \frac{n}{\omega_{n-1}} \frac{\langle x-y, \nu(y)\rangle\left(x_{j}-y_{j}\right)\left(x_{k}-y_{k}\right)}{|x-y|^{n+2}} \\
& -L_{2}(r) \frac{1}{\omega_{n-1}} \frac{\left(x_{j}-y_{j}\right) \nu_{k}(y)-\left(x_{k}-y_{k}\right) \nu_{j}(y)}{|x-y|^{n}}
\end{aligned}
$$

where

$$
L_{1}(r):=\frac{\mu\left(2 \mu+\mu^{\prime}\right)-r \mu^{\prime}}{2 \mu\left(\mu+\mu^{\prime}\right)}, \quad L_{2}(r):=\frac{\mu \mu^{\prime}-r\left(2 \mu+\mu^{\prime}\right)}{2 \mu\left(\mu+\mu^{\prime}\right)} .
$$

It is here that the usefulness of making a judicious choice for the parameter $r$ is most apparent. Specifically, for

$$
r:=\frac{\mu \mu^{\prime}}{2 \mu+\mu^{\prime}}
$$


we have $L_{2}(r)=0$ and, hence, the last term in (4.13) drops out. Consequently, the operator (4.13) corresponding to the choice (4.15), referred to in the literature as the pseudo-stress elastic double layer (cf., e.g., [15]), takes the form (4.5). We shall denote this operator by $K_{\psi}$.

In summary, for the choice of the parameter $r$ as in (4.15), the operator (4.12) becomes of the type (4.5). Hence,

$$
\nu \in \operatorname{VMO}(\partial \Omega) \Longrightarrow K_{\psi}: L^{q}\left(\partial \Omega ; \mathbb{R}^{n}\right) \rightarrow L^{q}\left(\partial \Omega ; \mathbb{R}^{n}\right)
$$

is a compact operator for every index $q \in(1, \infty)$.

Extending this compactness property to the scale of boundary Besov spaces is done using the following remarkable one-sided compactness property for the real method of interpolation for (compatible) Banach couples proved by M. Cwikel in [5]:

Theorem 4.3. Assume that $X_{j}, Y_{j}, j=0,1$, are two compatible Banach couples and suppose that the linear operator $T: X_{j} \rightarrow Y_{j}$ is bounded for $j=0$ and compact for $j=1$. Then the operator $T:\left(X_{0}, X_{1}\right)_{\theta, p} \rightarrow\left(Y_{0}, Y_{1}\right)_{\theta, p}$ is compact for all $\theta \in(0,1)$ and $p \in[1, \infty]$.

Granted (4.16), this theorem (used in the current context with $X_{0}:=L^{q}\left(\partial \Omega ; \mathbb{R}^{n}\right)$ and $X_{1}:=W^{1, q}\left(\partial \Omega ; \mathbb{R}^{n}\right)$, the vector-valued version of the $L^{q}$-based Sobolev space of order one on $\partial \Omega$ ) shows that

$$
\begin{gathered}
K_{\psi} \text { is compact on } B_{s}^{q, p}\left(\partial \Omega ; \mathbb{R}^{n}\right) \text { for all } 1<p, q<\infty \\
\text { and } 0<s<1 \text {, granted that } \nu \in \operatorname{VMO}(\partial \Omega) .
\end{gathered}
$$

Moving on, we may conclude that the problem (4.1) is well-posedness as soon as we show that the operator

$$
\begin{aligned}
& \mathcal{A}: W^{1, q}\left(\Omega ; \mathbb{R}^{n}\right) \rightarrow W^{-1, q}\left(\Omega ; \mathbb{R}^{n}\right) \oplus B_{1-\frac{1}{q}}^{q, q}\left(\partial \Omega ; \mathbb{R}^{n}\right), \\
& \mathcal{A} u:=\left(-\mu \Delta u-\mu^{\prime} \nabla \operatorname{div} u, \operatorname{Tr} u\right), \quad \forall u \in W^{1, q}\left(\Omega ; \mathbb{R}^{n}\right),
\end{aligned}
$$

is an isomorphism, where $\operatorname{Tr}: W^{1, q}\left(\Omega ; \mathbb{R}^{n}\right) \rightarrow B_{1-\frac{1}{q}}^{q, q}\left(\partial \Omega ; \mathbb{R}^{n}\right)$ denotes the boundary trace operator. In this vein, the hardest part is proving that the operator in question has closed range, of finite co-dimension. To address this issue, denote by $\Pi$ the volume potential given by

$$
\Pi w(x):=\int_{\Omega} E(x-y) w(y) d y, \quad x \in \Omega .
$$

This is smoothing of order two, hence maps $W^{-1, q}\left(\Omega ; \mathbb{R}^{n}\right)$ boundedly into $W^{1, q}\left(\Omega ; \mathbb{R}^{n}\right)$. Given that for every $g$ we have $\left(-\mu \Delta-\mu^{\prime} \nabla \operatorname{div}\right) \mathcal{D}_{r} g=0$ in $\Omega$, and that for every $q \in(1, \infty)$ and every $r \in \mathbb{R}^{n}$ the operator $\mathcal{D}_{r}$ maps $B_{1-\frac{1}{q}}^{q, q}\left(\partial \Omega ; \mathbb{R}^{n}\right)$ boundedly into $W^{1, q}\left(\Omega ; \mathbb{R}^{n}\right.$ ) (cf. [18] for much more general results of this flavor), it follows that the range of $\mathcal{\mathcal { A }}$ from (4.18) contains the subspace

$$
\begin{aligned}
& \left\{\left(-\mu \Delta u-\mu^{\prime} \nabla \operatorname{div} u, \operatorname{Tr} u\right): u=\Pi f+\mathcal{D}_{r} g,\right. \\
& \left.\qquad f \in W^{-1, q}\left(\Omega ; \mathbb{R}^{n}\right), g \in B_{1-\frac{1}{q}}^{q, q}\left(\partial \Omega ; \mathbb{R}^{n}\right)\right\} \\
& =\left\{f, \operatorname{Tr}(\Pi f)+\left(\frac{1}{2} I+K_{\psi}\right) g: f \in W^{-1, q}\left(\Omega ; \mathbb{R}^{n}\right), g \in B_{1-\frac{1}{q}}^{q, q}\left(\partial \Omega ; \mathbb{R}^{n}\right)\right\} .
\end{aligned}
$$


However, thanks to (4.17), it is not difficult to check that the assignment

$$
(f, g) \mapsto\left(f, \operatorname{Tr}(\Pi f)+\left(\frac{1}{2} I+K_{\psi}\right) g\right)
$$

from $W^{-1, q}\left(\Omega ; \mathbb{R}^{n}\right) \oplus B_{1-\frac{1}{q}}^{q, q}\left(\partial \Omega ; \mathbb{R}^{n}\right)$ into itself is Fredholm (with index zero, in fact). In particular, it has a closed range, of finite co-dimension, and we conclude from this and the fact that the range of $\mathcal{A}$ contains the subspace described in the last line of (4.20) that the operator (4.18) also has a closed range, of finite co-dimension. With this in hand, the reminder of the proof of the invertibility of $\mathcal{A}$ is routine, so we omit it. Ultimately, the conclusion is that, if $\nu \in \operatorname{VMO}(\partial \Omega)$ then the Poisson problem

$$
\left\{\begin{array}{l}
\mu \Delta u+\mu^{\prime} \nabla \operatorname{div} u=f \in W^{-1, q}\left(\Omega ; \mathbb{R}^{n}\right), \\
\operatorname{Tr} u=g \in B_{1-\frac{1}{q}}^{q, q}\left(\partial \Omega ; \mathbb{R}^{n}\right), \\
u \in W^{1, q}\left(\Omega ; \mathbb{R}^{n}\right),
\end{array}\right.
$$

is well-posed for every $q \in(1, \infty)$. Finally, taking $g=0$ then yields the conclusion in item (ii) of Theorem 1.1 .

In closing, we wish to note that the (infinitesimal, mean) oscillation of the unit normal, quantified as

$$
\{\nu\}_{\text {Osc }(\partial \Omega)}:=\lim _{\varepsilon \rightarrow 0}\left(\sup _{B_{\varepsilon}} f_{B_{\varepsilon} \cap \partial \Omega} f_{B_{\varepsilon} \cap \partial \Omega}|\nu(x)-\nu(y)| d \sigma(x) d \sigma(y)\right)
$$

where the supremum is taken over the collection $\left\{B_{\varepsilon}\right\}$ of disks with centers on $\partial \Omega$ and of radius $\leq \varepsilon$, is actually equivalent to the distance of $\nu$ to $\operatorname{VMO}(\partial \Omega)$. That is,

$$
\{\nu\}_{\mathrm{Osc}(\partial \Omega)} \approx \operatorname{dist}(\nu \operatorname{VMO}(\partial \Omega)) .
$$

This observation allows for a more intuitive interpretation of the condition (4.2). As an example, consider the case when $\Omega$ is a curvilinear polygon with precisely one angular point located at the origin $0 \in \mathbb{R}^{2}$. Furthermore, assume that, in a neighborhood of $0, \partial \Omega$ agrees with a sector of aperture $\theta \in(0, \pi)$ with vertex at 0 . In particular, the outward unit normal $\nu$ to $\Omega$ is smooth on $\partial \Omega \backslash\{0\}$ and is piecewise constant near 0 , where it assumes two values, say, $\nu_{+}$and $\nu_{-}$. As a result,

$$
\{\nu\}_{\mathrm{Osc}(\partial \Omega)} \approx\left\|\nu_{+}-\nu_{-}\right\| \approx \sqrt{1+\cos \theta},
$$

which shows that the condition (4.2) amounts, in this case, to the requirement that the angle $\theta$ is sufficiently close to $\pi$.

\section{References}

[1] W. ARENDt. Semigroups and evolution equations: functional calculus, regularity and kernel estimates. Evolutionary equations, Vol. I, pp. 1-85, Handb. Differ. Equ., North-Holland, Amsterdam, 2004.

[2] P. Auscher, S. Hofmann, M. Lacey, A. Mcintosh, P. Tchamitchian. The solution of the Kato square root problem for second order elliptic operators in $\mathbb{R}^{n}$. Ann. of Math., 156 (2002), 633-654. 
[3] F. Bernicot, J. Zhao. On maximal $L^{p}$-regularity. J. Funct. Anal., 256 (2009), 2561-2586.

[4] R. Coifman, G. Weiss. Extensions of Hardy spaces and their use in analysis. Bull. Amer. Math. Soc. 83 (1977), no. 4, 569-645.

[5] M. Cwikel. Real and complex interpolation and extrapolation of compact operators. Duke Math. J., 65 (1992), no. 2, 333-343.

[6] R. Danchin. On the solvability of the compressible Navier-Stokes system in bounded domains. Preprint, (2009).

[7] P. Hajlasz. Sobolev spaces on an arbitrary metric space. Potential Anal., 5 (1996), no. 4, 403-415.

[8] S. Hofmann, M. Mitrea and M. Taylor. Singular integrals and elliptic boundary problems on regular Semmes-Kenig-Toro domains. Preprint, (2008).

[9] D. Jerison, C. Kenig. Boundary behavior of harmonic functions in nontangentially accessible domains. Adv. in Math. 46 (1982), no. 1, 80-147.

[10] P.W. Jones. Quasiconformal mappings and extendability of functions in Sobolev spaces. Acta Math., 147 (1981), no. 1-2, 71-88.

[11] A. Jonsson, H. Wallin. Function spaces on subsets of $\mathbb{R}^{n}$. Math. Rep., 2 (1984), no. 1, xiv+221 pp.

[12] C.E. Kenig. Harmonic Analysis Techniques for Second Order Elliptic Boundary Value Problems, AMS CBMS Vol. 83, 1984.

[13] P.C. Kunstmann. On maximal regularity of type $L^{p}-L^{q}$ under minimal assumptions for elliptic non-divergence operators. J. Funct. Anal., 255 (2008), $2732-2759$.

[14] P.C. Kunstmann And L. Weis. Maximal $L^{p}$-regularity for parabolic equations, Fourier multiplier theorems and $H^{\infty}$-functional calculus. Functional analytic methods for evolution equations, pp. 65-311, Lecture Notes in Math., Vol. 1855, Springer, Berlin, 2004.

[15] V.D. Kupradze. Potential Methods in the Theory of Elasticity, Daniel Davey \& Co., Inc., New York 1965.

[16] O.A. LAdyzhenskayA. The Mathematical Theory of Viscous Incompressible Flow, Gordon and Breach Science Publishers, New York-London, 1963.

[17] S. Mayboroda, M. Mitrea. The Poisson problem for the Lamé system on low-dimensional Lipschitz domains. Integral methods in science and engineering, 137-160, Birkhäuser Boston, Boston, MA, 2006.

[18] D. Mitrea, M. Mitrea and S. Monniaux. Singular integrals on a class of Ahlfors-David domains and the Dirichlet problem with data in Besov spaces. Preprint, (2009).

[19] M. Mitrea, S. Monniaux. On the analyticity of the semigroup generated by the Stokes operator with Neumann type boundary conditions on Lipschitz subdomains of Riemannian manifolds. Trans. Amer. Math. Soc., 361 (2009), $3125-3157$.

[20] M. Mitrea, M. Wright. Boundary Value Problems for the Stokes System in arbitrary Lipschitz Domains. Preprint, (2008).

[21] M. Mitrea, M. Wright. Transmission problems for the Lamé system in Lipschitz domains. Preprint, (2009). 
[22] S. Monniaux. Maximal regularity and appications to PDE's, to appear in Analytical and Numerical Aspects of Partial Differential Equations, Notes of a Lecture Series, E. Emmrich and P. Wittbold eds., Walter de Gruyter, Proceedings in Mathematics, ISBN 978-3-11-020447-6, 2009.

[23] Z. Shen. Resolvent estimates in $L^{p}$ for elliptic systems in Lipschitz domains. J. Funct. Anal., 133 (1995), no. 1, 224-251.

Marius Mitrea

Department of Mathematics University of Missouri-Columbia Mathematical Sciences Building Columbia, MO 65211, USA

e-mail: mitream@missouri.edu
Sylvie Monniaux

LATP - UMR 6632 - Case Cour A

Faculté des Sciences de Saint-Jérôme

Université Aix-Marseille 3

F-13397 Marseille Cédex 20, France

e-mail: sylvie.monniaux@univ-cezanne.fr 